\title{
Acute effects of bronchial thermoplasty: a matter of concern or an indicator of possible benefit to small airways?
}

\author{
Louis-Philippe Boulet and Michel Laviolette
}

\begin{abstract}
Affiliation: Institut universitaire de cardiologie et de pneumologie de Québec - Université Laval (IUCPQ-UL), Québec, QC, Canada.

Correspondence: $\operatorname{Dr}$ Louis-Philippe Boulet, Institut universitaire de cardiologie et de pneumologie de Québec - Université Laval, 2725, Chemin Ste-Foy, Québec, QC, Canada G1V 4G5. E-mail: Ipboulet@amed.ulaval.ca
\end{abstract}

@ERSpublications

Acute effects of bronchial thermoplasty on small airways and lung parenchyma: good or bad? http://ow.ly/T6Uu309keuf

Cite this article as: Boulet L-P, Laviolette M. Acute effects of bronchial thermoplasty: a matter of concern or an indicator of possible benefit to small airways? Eur Respir J 2017; 49: 1700029 [https://doi.org/10.1183/ 13993003.00029-2017].

Bronchial thermoplasty (BT) is a non-pharmacological treatment developed in the last two decades that has been proposed for the treatment of difficult-to-control asthma despite current standard asthma treatment [1-3]. This technique consists of delivering temperature-controlled radio-frequency energy to bronchi from about 3 to $10 \mathrm{~mm}$ in diameter through an endoscope. The initial concept underlying this technique was to improve airway function in decreasing airway smooth mass in the treated area, as shown in animal models and in a study of subjects undergoing lung surgery for cancer $[4,5]$.

The randomised controlled trials performed on BT showed that it could improve asthma-related quality of life as well as reduce severe exacerbations, absenteeism from work/school and healthcare use [6-8]. On the other hand, during the days following the procedure, patients usually had a variable increase in their asthma symptoms, which could require treatment with oral corticosteroids and, in some, hospital admission, mostly in those with very severe asthma.

The first assessments of this technique raised concerns about its potential detrimental long-term effects, particularly on the integrity of the bronchial wall. However, 5-year follow-up of these clinical trial cohorts showed that BT resulted in persistent asthma control improvements, with stable forced expiratory volume in $1 \mathrm{~s}\left(\mathrm{FEV}_{1}\right)$ and no significant bronchial structural abnormalities on high-resolution computed tomography (CT) scan, except for $3 \%$ of patients who had increased or new bronchiectasis at 5 years compared with the baseline, although not necessarily because of BT [9-11]. Overall, these studies demonstrated the efficacy and safety of BT in subjects with persistent asthma.

In this issue of the European Respiratory Journal, DEBRAY et al. [12] report CT findings in 13 patients after BT for treatment of severe unstable asthma. The day after treatment, patients presented peri-bronchial consolidations and ground-glass opacities without symptoms or signs of infection. Involvement of the lobes adjacent to the treated lobe was observed in $32 \%$ of BT treatments. Pleural effusion or fissure thickening and septal thickening were also observed in $68 \%$ and $39 \%$ of BT treatments respectively. These results show a high incidence of tissue alterations extended to the peripheral lung located over the treated lobes and reaching pleura, interlobar fissure and adjacent lobes. These modifications occurred in patients who had received oral prednisone $50 \mathrm{mg} \cdot \mathrm{day}^{-1}$ for 3 days and who presented with no clinically significant adverse events. CT

Received: Jan 062017 | Accepted: Jan 092017

Conflict of interest: Disclosures can be found alongside this article at erj.ersjournals.com

Copyright CERS 2017 
abnormalities decreased at 1 week and had resolved 1 month after the procedure in 10 of 15 treated lobes, and decreased in the five others. A few months later, three treated lobes showed mild focal bronchial dilatation.

These findings are certainly of interest and may explain, at least partly, the short-term worsening of respiratory conditions after this procedure in some patients. These changes could possibly increase ventilation/perfusion defects, reduce lung compliance and increase the work of breathing, contributing to the short-term increase in respiratory symptoms after the procedure. It is however reassuring that they resolved in the majority of patients and did not result in permanent defects, in keeping with previous reports.

In regard to the mechanisms underlying their observations, DeBray et al. [12] reported no histological analysis and postulated that these alterations "reflect alveolar inflammation and oedema" due to heat-shock injury induced by BT-induced heat reaching small airways and lung parenchyma.

In the previous study on an animal model, one of two dogs killed 1 week after BT showed bronchial wall thickening on CT and histological analysis of these airways suggested that it resulted from oedema at the treated area [4]. No significant parenchymal oedema was found and no significant radiographic abnormalities were observed in dogs killed at 6, 12 and 157 weeks post BT. In the first study in humans, six subjects scheduled for lung surgery were treated with $65^{\circ} \mathrm{C}$ activations. Histological analyses performed 1-3 weeks post treatment showed in some tissue sections necrosis of mucus ducts and glands, thrombosis in perichondral vessels, mild focal necrotic cartilage or peribronchial pneumonitis characterised by patchy accumulation of inflammatory cells, mostly lymphocytes in interstitial spaces [5].

Histological analyses of BT effects on asthmatic airways have been recently published. They were limited to small bronchial samples obtained from bronchoscopies. They showed that BT decreased airway smooth muscle mass, ASM-associated nerves and neuroendocrine epithelial cells and reticular basement membrane collagen depositions $[13,14]$ and that the changes in ASM mass and collagen deposition persisted 27-48 months after BT treatment [15].

The occurrence of transient reversible pulmonary opacities following BT treatment may suggest that BT-induced heat can reach small airways and lung parenchyma and that these effects induce changes in these areas. Consequently, it is not excluded that BT alters small airways and lung parenchyma in a way to improve asthma control. On the other hand, the Debray et al. observations raise the question whether we should try to prevent the observed acute changes more effectively to avoid or minimise the observed transient increase in respiratory symptoms post BT. Out of 558 bronchial thermoplasty procedures of the Air 2 study performed in 190 subjects [7], there was an increased incidence of transient increase in asthma symptom worsening, upper and lower respiratory tract infection atelectasis and hospitalisations in the BT group compared with the sham group. The median time to onset of these respiratory events after the procedure was 1 day resolving within 1 week.

We believe that the occurrence of transient reversible pulmonary opacities following BT treatment suggest either that thermal effects of BT reached the small airways and alveolar area, causing local inflammation and oedema as previously observed in treated controls scheduled for lung surgery [5], or that the BT effects on treated airways extend deeply enough into the bronchial wall to induce oedema and exudation that reaches small airways and alveolar area by extravasation. Both hypotheses could be supported by the observation that the extent of the peribronchial consolidations is proportional to the number of heat activations and, consequently, to the amount of heat energy applied to the area. In the absence of histological analysis, it will be difficult to resolve these issues.

In conclusion, Debray et al. [12] report new data on the acute effects of BT. We may question the need to find interventions helping to prevent these acute events in order to reduce acute symptoms post procedure or potential long-term detrimental effects although these last seem limited and of doubtful long-term clinical significance. We may ask if extended effects of BT to parenchyma induce structural changes at the small airway level that contribute to asthma control improvement [16]. Histological and CT analyses of these areas in an animal model [4] or patients scheduled for lung surgery [5] or less invasive techniques such as optical coherence tomography in asthmatics [17] a few days to months after BT treatment when possible would help investigate the nature of the observed changes on high-resolution CT and their long-term consequences on small airways and parenchymal anatomy, as well as on asthma control.

\section{References}

1 Dombret MC, Alagha K, Boulet LP, et al. Bronchial thermoplasty: a new therapeutic option for the treatment of severe, uncontrolled asthma in adults. Eur Respir Rev 2014; 23: 510-518.

2 Castro M, Musani AI, Mayse ML, et al. Bronchial thermoplasty: a novel technique in the treatment of severe asthma. Ther Adv Respir Dis 2010; 4: 101-116.

3 Panettieri RA Jr. Bronchial Thermoplasty: Targeting Structural Cells in Severe Persistent Asthma. Ann Am Thorac Soc 2015; 12: 1593-1594.

4 Danek CJ, Lombard CM, Dungworth DL, et al. Reduction in airway hyperresponsiveness to methacholine by the application of RF energy in dogs. J Appl Physiol 2004; 97: 1946-1953. 
5 Miller JD, Cox G, Vincic L, et al. A prospective feasibility study of bronchial thermoplasty in the human airway. Chest 2005; 127: 1999-2006.

6 Cox G, Thomson NC, Rubin AS, et al. Asthma control during the year after bronchial thermoplasty. N Engl J Med 2007; 356: 1327-1337.

7 Castro M, Rubin AS, Laviolette M, et al. Effectiveness and safety of bronchial thermoplasty in the treatment of severe asthma: a multicenter, randomized, double-blind, sham-controlled clinical trial. Am J Respir Crit Care Med 2010; 181: 116-124.

8 Pavord ID, Cox G, Thomson NC, et al. Safety and efficacy of bronchial thermoplasty in symptomatic, severe asthma. Am J Respir Crit Care Med 2007; 176: 1185-1191.

9 Thomson NC, Rubin AS, Niven RM, et al. Long-term (5 year) safety of bronchial thermoplasty: Asthma Intervention Research (AIR) trial. BMC Pulm Med 2011; 11: 8.

10 Wechsler ME, Laviolette M, Rubin AS, et al. Bronchial thermoplasty: long-term safety and effectiveness in patients with severe persistent asthma. I Allergy Clin Immunol 2013; 132: 1295-1302.

11 Pavord ID, Thomson NC, Niven RM, et al. Safety of bronchial thermoplasty in patients with severe refractory asthma. Ann Allergy Asthma Immunol 2013; 111: 402-407.

12 Debray MP, Dombret MC, Pretolani M, et al. Early computed tomography modifications following bronchial thermoplasty in patients with severe asthma. Eur Respir J 2017; 49: 1601565.

13 Chakir J, Haj-Salem I, Gras D, et al. Effects of bronchial thermoplasty on airway smooth muscle and collagen deposition in asthma. Ann Am Thorac Soc 2015; 12: 1612-1618.

14 Pretolani M, Dombret MC, Thabut G, et al. Reduction of airway smooth muscle mass by bronchial thermoplasty in patients with severe asthma. Am J Respir Crit Care Med 2014; 190: 1452-1454.

15 Haj-Salem I, Boulet LP, Biardel S, et al. Long-term effects of bronchial thermoplasty on airway smooth muscle and reticular basement membrane thickness in severe asthma. Ann Am Thorac Soc 2016; 13: 1426-1428.

16 Contoli M, Kraft M, Hamid Q, et al. Do small airway abnormalities characterize asthma phenotypes? In search of proof. Clin Exp Allergy 2012; 42: 1150-1160.

17 Kirby M, Ohtani K, Lopez Lisbona RM, et al. Bronchial thermoplasty in asthma: 2-year follow-up using optical coherence tomography. Eur Respir J 2015; 46: 859-862. 